\title{
Stochastic pumping of heat: Approaching the Carnot efficiency
}

\author{
Dvira Segal \\ Chemical Physics Theory Group, Department of Chemistry, \\ University of Toronto, 80 St. George Street, Toronto, Ontario M5S 3H6, Canada
}

(Dated: November 2, 2018)

\begin{abstract}
Random noise can generate a unidirectional heat current across asymmetric nano objects in the absence (or against) a temperature gradient. We present a minimal model for a molecularlevel stochastic heat pump that may operate arbitrarily close to the Carnot efficiency. The model consists a fluctuating molecular unit coupled to two solids characterized by distinct phonon spectral properties. Heat pumping persists for a broad range of system and bath parameters. Furthermore, by filtering the reservoirs' phonons the pump efficiency can approach the Carnot limit.
\end{abstract}

PACS numbers: $\quad$ 05.40.-a 63.22.-m, 44.10.+i 05.70.Ln

Heat flows spontaneously from objects at high temperature to objects of low temperature. Directing heat against a temperature gradient requires the application of an external perturbation or the design of a special system-bath initial condition. A generic nano-scale heat pump model includes a molecular element bridging two solids. An external force modulates the subsystem (molecular) energetics, leading to a net exchange of heat between the baths against, or in the absence, of a temperature gradient. A spatial asymmetry should be built into the system in order to define a preferential directionality. This process is closely related to the ratchet effect where a particle current is catalyzed in the absence of a dc voltage drop due to the asymmetry in the spatial potential and the influence of time dependent forces [1].

Recent studies have carefully analyzed the operation principles of prototype classical [2, 3, ,4] and quantum 5, 6, 7, 8, 9, 10, 11] thermal machines, seeking to optimize performance. Typically, in these schemes a carefully-shaped external force periodically modulates the levels of the nano object, leading to the pumping operation. In electronic systems a Brownian electron refrigerator based on selective tunneling through a metalinsulator-superconductor junction was recently proposed [12]. Fluctuating electric fields can also do chemical work, driving ions against an electrochemical potential difference [13].

In this paper we demonstrate facile pumping of heat in the form of vibrational energy in response to random noise. The following generic effect is discussed: Heat can be pumped between distinct solids when the bridging object suffers stochastic fluctuations of its energies, given that the spectral density of at least one solid strongly varies within the noise spectral window. Furthermore, using reservoirs with suitable energy filters, (Einstein solids) one can construct a heat pump that works close to the Carnot limit. Thus, we demonstrate that not only can one utilize random noise and pump heat against a bias ("Maxwell demon"), the efficiency of this machine

APS copyright, to be printed in Phys. Rev. Lett. may approach the maximal value.

Our minimal model consists a Kubo oscillator [14] bilinearly coupled to two macroscopic reservoirs. The model could be realized by adsorbing a molecule on a dielectric surface, blocking charge transfer, while allowing for heat exchange between the modules. The total Hamiltonian includes the following contributions, $H=H_{0}(t)+H_{B}+V_{L}+V_{R} . H_{0}$ includes the isolated subsystem, a Kubo oscillator,

$H_{0}(t)=\sum_{n=0,1 . . \infty} E_{n}(t)|n\rangle\langle n| ; \quad E_{n}(t)=\left[E_{n}^{(0)}+\epsilon_{n}(t)\right] .(1)$

$E_{n}^{(0)}=n B_{0}$ is the static energy of level $|n\rangle, \epsilon_{n}(t)$ is a time dependent stochastic contribution. We assume that the average over stationary fluctuations vanishes, $\langle\epsilon(t)\rangle_{\epsilon}=0$, while higher moments survive. This local oscillator is coupled to two thermal baths of temperatures $T_{\nu}=1 / \beta_{\nu}$ $\left(k_{B} \equiv 1\right)$, represented by sets of independent harmonic oscillators $(\hbar \equiv 1)$

$$
H_{B}=\sum_{\nu, k} \omega_{k} b_{\nu, k}^{\dagger} b_{\nu, k} .
$$

$b_{\nu, k}^{\dagger}, b_{\nu, k}$ are the bosonic creation and annihilation operators respectively for the mode $k$ of bath $\nu=L, R$. The interaction between the subsystem and the bath is taken to be bilinear (displacement-displacement) [15],

$$
\begin{aligned}
V_{\nu} & =F_{\nu} \sum_{n=1,2 . . \infty} c_{n}|n\rangle\langle n-1|+c . c . \\
F_{\nu} & =\sum_{k} \lambda_{\nu, k}\left(b_{\nu, k}^{\dagger}+b_{\nu, k}\right),
\end{aligned}
$$

where $c_{n}=\sqrt{n}$, and the system-bath interaction is characterized by the spectral function

$$
g_{\nu}(\omega)=2 \pi \sum_{\nu, k} \lambda_{\nu, k}^{2} \delta\left(\omega-\omega_{k}\right) .
$$

In what follows we simplify the discussion and consider a two-level system (TLS) $(n=0,1)$ for the local Hamiltonian,

$$
\begin{aligned}
H_{0}^{T L S}(t) & =B(t) \sigma_{z} / 2 ; \quad B(t)=\left[B_{0}+\epsilon(t)\right] \\
V_{\nu}^{T L S} & =F_{\nu} \sigma_{x} .
\end{aligned}
$$


Here $\sigma_{x}=|1\rangle\langle 0|+| 0\rangle\left\langle 1\left|, \sigma_{z}=\right| 1\right\rangle\langle 1|-| 0\rangle\langle 0|, B_{0}=E_{1}^{(0)}-$ $E_{0}^{(0)}$, and $\epsilon(t)$ is the stochastic modulation of this energy gap. We sometimes refer to the truncated harmonic mode as a "spin". In the weak coupling limit the TLS dynamics can be trivially carried back to the harmonic limit $(n=$ $0,1, \ldots \infty)$, as we discuss below.

Stochastically averaged master equation. Closed kinetic equations for the $|n\rangle$ state population can be obtained in the weak system-bath coupling limit [16]. Briefly, starting with the quantum Liouville equation, this involves (i) studying the quantum dynamics within second order perturbation theory, (ii) going into the markovian limit, assuming short correlation time of bath fluctuations, and (ii) averaging over the stochastic process under the decoupling approximation,

$$
\left\langle e^{i \int_{0}^{x} \epsilon\left(t^{\prime}\right) d t^{\prime}} P_{n}(x)\right\rangle_{\epsilon} \sim\left\langle e^{i \int_{0}^{x} \epsilon\left(t^{\prime}\right) d t^{\prime}}\right\rangle_{\epsilon}\left\langle P_{n}(x)\right\rangle_{\epsilon} .
$$

Here $P_{n}(n=0,1)$ denotes the TLS population, and the average is performed over energy fluctuations. The decomposition (6) relays on the separation of timescales: Energy fluctuations are assumed to be fast in comparison to the characteristic subsystem relaxation time. The three assumptions (i)-(iii) result in the following equations $\left\langle P_{1}(t)\right\rangle_{\epsilon}+\left\langle P_{0}(t)\right\rangle_{\epsilon}=1$,

$$
\begin{aligned}
\left\langle\dot{P}_{1}(t)\right\rangle_{\epsilon} & =-\left(k_{1 \rightarrow 0}^{L}+k_{1 \rightarrow 0}^{R}\right)\left\langle P_{1}(t)\right\rangle_{\epsilon} \\
& +\left(k_{0 \rightarrow 1}^{L}+k_{0 \rightarrow 1}^{R}\right)\left\langle P_{0}(t)\right\rangle_{\epsilon},
\end{aligned}
$$

with the activation and relaxation rates

$$
\begin{aligned}
& k_{0 \rightarrow 1}^{\nu}=\int_{-\infty}^{\infty} d \omega g_{\nu}(\omega) N_{\nu}(\omega) I\left(B_{0}-\omega\right), \\
& k_{1 \rightarrow 0}^{\nu}=\int_{-\infty}^{\infty} d \omega g_{\nu}(\omega)\left[N_{\nu}(\omega)+1\right] I\left(B_{0}-\omega\right) .
\end{aligned}
$$

Here $g_{\nu}(\omega)$ is the phonon spectral function defined in (4), extended to negative values $g_{\nu}(\omega)=-g_{\nu}(-\omega), N_{\nu}(\omega)=$ $\left[e^{\beta_{\nu} \omega}-1\right]^{-1}$ is the Bose-Einstein occupation factor and

$$
I(\omega)=\frac{1}{2 \pi} \int_{-\infty}^{\infty} d t e^{i \omega t}\left\langle e^{i \int_{0}^{t} \epsilon\left(t^{\prime}\right) d t^{\prime}}\right\rangle_{\epsilon}
$$

is the spectral lineshape of the Kubo oscillator. Assuming a Gaussian process, going to the fast modulation limit $\left\langle\epsilon\left(t_{1}\right) \epsilon\left(t_{2}\right)\right\rangle=2 \gamma \delta\left(t_{1}-t_{2}\right)$, the noise spectral lineshape can be approximated by a Lorentzian function

$$
I(\omega)=\frac{\gamma / \pi}{\omega^{2}+\gamma^{2}}
$$

of width $\gamma$. For $\gamma \rightarrow 0, I(\omega)=\delta(\omega)$, and the field-free vibrational relaxation rates [17] are retrieved from Eq. (8): The rates are evaluated at the bare TLS frequency, $k_{0 \rightarrow 1}^{\nu}=g_{\nu}\left(B_{0}\right) N_{\nu}\left(B_{0}\right), k_{1 \rightarrow 0}^{\nu}=g_{\nu}\left(B_{0}\right)\left[N_{\nu}\left(B_{0}\right)+1\right]$, obeying the detailed balance condition. In contrast, for a general driving this condition is violated, as the time dependent field drives the subsystem out of thermal equilibrium with the bath.
The master equation (7) can be trivially generalized in the weak coupling regime to describe a harmonic local mode [Eqs. (11)-(3)] under a stochastic field,

$$
\begin{aligned}
& \left\langle\dot{P}_{n}\right\rangle_{\epsilon}=-\sum_{\nu}\left[(n+1) k_{n \rightarrow n+1}^{\nu}+n k_{n \rightarrow n-1}^{\nu}\right]\left\langle P_{n}\right\rangle_{\epsilon} \\
& +(n+1) \sum_{\nu} k_{n+1 \rightarrow n}^{\nu}\left\langle P_{n+1}\right\rangle_{\epsilon}+n \sum_{\nu} k_{n-1 \rightarrow n}^{\nu}\left\langle P_{n-1}\right\rangle_{\epsilon} .
\end{aligned}
$$

Note that for harmonic systems the microscopic rates are independent of the index $n$, thus we only identify activation and relaxation processes using (8).

Heat current. We derive an analytical expression for the heat flux in the presence of stochastic energy modulations. We begin with the TLS model (5), then extend the results to the harmonic case. An expression for the energy flux operator, applicable in both stationary and time dependent situations, was given in [18], $\hat{J}_{R}=\frac{i}{2}\left[\left(H_{0}^{T L S}-H_{B}\right), V_{R}^{T L S}\right]$. The current is defined positive when flowing from $L$ to $R$, see Fig. 11 We substitute Eqs. (2) and (5) into this expression and obtain

$$
\hat{J}_{R}(t)=-\frac{1}{2}\left[\sigma_{x} G_{R}+B(t) \sigma_{y} F_{R}\right]
$$

with $\sigma_{y}=-i|1\rangle\langle 0|+i| 0\rangle\langle 1|, F_{\nu}=\sum_{k} \lambda_{\nu, k}\left(b_{\nu, k}^{\dagger}+b_{\nu, k}\right)$, $G_{\nu}=i \sum_{k} \lambda_{\nu, k} \omega_{k}\left(b_{\nu, k}^{\dagger}-b_{\nu, k}\right)$. The expectation value of the current is given by $J=\operatorname{Tr}\{\rho \hat{J}\}$, where $\rho$ is the total density matrix, and the trace is performed over the thermal bath and the subsystem degrees of freedom. A further averaging over the TLS energy fluctuations is necessary. Since in steady-state the condition $\left\langle\operatorname{Tr}\left\{\partial V_{R} / \partial t\right\}\right\rangle_{\epsilon}=0$ translates to $\left\langle\operatorname{Tr}\left\{G_{R} \sigma_{x}\right\}\right\rangle_{\epsilon}=$ $\left\langle\operatorname{Tr}\left\{B(t) \sigma_{y} F_{R}\right\}\right\rangle_{\epsilon}$, the stationary noise-averaged current can be calculated by studying the first term in (12), $\left\langle J_{R}\right\rangle_{\epsilon}=-\left\langle\operatorname{Tr}_{B}\left\{\rho_{1,0} G_{R}+\rho_{0,1} G_{R}\right\}\right\rangle_{\epsilon}$. Here $\rho_{i, j}$ are the matrix elements of the density matrix, and $\operatorname{Tr}_{B}$ denotes a trace over the bath states. Next, using a second order expansion of $\rho_{1,0}$, adopting the same set of approximations employed above for deriving (7), we acquire the steady-state heat current expression

$$
\left\langle J_{R}\right\rangle_{\epsilon}=\left\langle P_{1}\right\rangle_{\epsilon} f_{1 \rightarrow 0}^{R}-\left\langle P_{0}\right\rangle_{\epsilon} f_{0 \rightarrow 1}^{R} .
$$

The noise-averaged population is obtained by solving (7) in steady-state, taking $\left\langle\dot{P}_{n}\right\rangle_{\epsilon}=0$. The energy absorption and dissipation rates (Energy/time) are given by

$$
\begin{aligned}
& f_{0 \rightarrow 1}^{\nu}=\int_{-\infty}^{\infty} d \omega \omega g_{\nu}(\omega) I\left(B_{0}-\omega\right) N_{\nu}(\omega) \\
& f_{1 \rightarrow 0}^{\nu}=\int_{-\infty}^{\infty} d \omega \omega g_{\nu}(\omega) I\left(B_{0}-\omega\right)\left[N_{\nu}(\omega)+1\right]
\end{aligned}
$$

Note that in the absence of fluctuations the rates satisfy the relation $f_{n \rightarrow n \pm 1}^{\nu}=B_{0} k_{n \rightarrow n \pm 1}^{\nu}$ [17], while in the presence of stochastic force we cannot trivially connect the energy transition rates (14) to the population relaxation rates (8). In the weak coupling limit Eq. (13) 
can be easily generalized to the harmonic (Kubo) case, $\left\langle J_{R}\right\rangle_{\epsilon}=\sum_{n=1}^{\infty} n\left[\left\langle P_{n}\right\rangle_{\epsilon} f_{n \rightarrow n-1}^{R}-\left\langle P_{n-1}\right\rangle_{\epsilon} f_{n-1 \rightarrow n}^{R}\right]$, with the population obtained by solving (11) in steady-state.

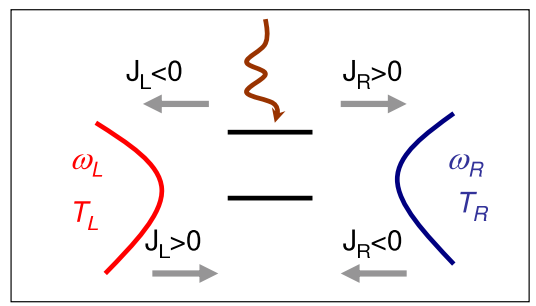

FIG. 1: Scheme of the stochastic pump. A molecular element under an external perturbation (curly line) is coupled to two solids of different spectral properties. The arrows display the sign notation.

Pumping mechanism. The pumping mechanism relays on two conditions: (i) The spectral function (of at least one bath) should strongly vary in the frequency window of the noise lineshape. (ii) The phonon reservoirs should have distinct spectral properties. We assume that $\omega_{R} \lesssim$ $B_{0}<\omega_{L} ; \omega_{\nu}$ is the $\nu$ contact cutoff vibrational frequency.

Due to the fast stochastic modulation, the spacing $B(t)$ can be abruptly reduced to values below $\omega_{R}$. Since these modulations are faster that the subsystem relaxation time, the TLS effective temperature becomes significantly low, even lower than $T_{R}$. The TLS then absorbs energy from both baths. In contrast, fluctuations that largely increase the gap lead to a high effective internal temperature, while practically disconnecting the $R$ bath from the system. If the internal temperature is higher than $T_{L}$, energy will dissipate from the Kubo mode to the left bath. Thus, in this asymmetric setup net energy can be injected from the $R$ bath to the $L$ bath even for $T_{L}>T_{R}$. Note that this principle is independent of the specific form assumed for the noise spectral function, the molecular properties, and the details of the baths.

This mechanism is reminiscent to the idea proposed in [10], with two crucial distinctions: (i) Here random perturbations catalyze (maybe even undesirably) heat flow, rather that carefully shaped pulses. (ii) The pump operates in the fast modulation regime, rather than adiabatically/quasiadibatically, leading to the Carnot efficiency under proper conditions.

An exactly solvable model. We employ next the Einstein model for the phonon spectral functions, $g_{\nu}(\omega)=$ $\xi_{\nu} \delta\left(\omega-\omega_{\nu}\right), \xi_{\nu}=2 \pi \lambda_{\nu}^{2}$ from (4). Practically, this scenario may be realized by filtering the contact's phonon modes [19]. Assuming that $I\left(B_{0}+\omega_{\nu}\right) \ll I\left(B_{0}-\omega_{\nu}\right)$ [20], the transition rates (8) and (14) reduce to

$$
\begin{aligned}
& k_{0 \rightarrow 1}^{\nu}=\xi_{\nu} I\left(B_{0}-\omega_{\nu}\right) N_{\nu}\left(\omega_{\nu}\right) \\
& k_{1 \rightarrow 0}^{\nu}=\xi_{\nu} I\left(B_{0}-\omega_{\nu}\right)\left[N_{\nu}\left(\omega_{\nu}\right)+1\right] \\
& f_{0 \rightarrow 1}^{\nu}=\omega_{\nu} \xi_{\nu} I\left(B_{0}-\omega_{\nu}\right) N_{\nu}\left(\omega_{\nu}\right) \\
& f_{1 \rightarrow 0}^{\nu}=\omega_{\nu} \xi_{\nu} I\left(B_{0}-\omega_{\nu}\right)\left[N_{\nu}\left(\omega_{\nu}\right)+1\right]
\end{aligned}
$$

and the energy current at the $\nu$ contact becomes 15]

$$
\left\langle J_{\nu}\right\rangle_{\epsilon}=\omega_{\nu} \mathcal{T}\left[N_{L}\left(\omega_{L}\right)-N_{R}\left(\omega_{R}\right)\right]
$$

where $\mathcal{T}=\frac{\gamma_{L}\left(\omega_{L}\right) \gamma_{R}\left(\omega_{R}\right)}{\gamma_{L}\left(\omega_{L}\right)+\gamma_{R}\left(\omega_{R}\right)}$ for the Hamiltonian (1)-(3) and $\mathcal{T}=\frac{\gamma_{L}\left(\omega_{L}\right) \gamma_{R}\left(\omega_{R}\right)}{\sum_{\nu} \gamma_{\nu}\left(\omega_{\nu}\right)\left[1+2 N_{\nu}\left(\omega_{\nu}\right)\right]}$ for the TLS model (5). Here $\gamma_{\nu}\left(\omega_{\nu}\right) \equiv \xi_{\nu} I\left(B_{0}-\omega_{\nu}\right)$. In both cases the $R$ contact is cooled down when $N_{R}\left(\omega_{R}\right)>N_{L}\left(\omega_{L}\right)$, i.e. for $\beta_{L} \omega_{L}>$ $\beta_{R} \omega_{R}$. Defining $\delta T=T_{L}-T_{R}>0, \delta \omega=\omega_{L}-\omega_{R}>0$, the pumping condition translates to

$$
\frac{\delta T}{T_{R}}<\frac{\delta \omega}{\omega_{R}} .
$$

For reversible systems the net current is zero, and an equality is attained, $\delta T / T_{R}=\delta \omega / \omega_{R}$. Note that for $\delta T=0$ a unidirectional current develops merely based on the asymmetry $\omega_{L}>\omega_{R}$. We calculate next the machine efficiency. In steady-state the work over time done by the external force is given by the difference $\langle W\rangle_{\epsilon}=\left\langle J_{R}\right\rangle_{\epsilon}-$ $\left\langle J_{L}\right\rangle_{\epsilon}$

$$
\langle W\rangle_{\epsilon}=\left(\omega_{R}-\omega_{L}\right) \mathcal{T}\left[N_{L}\left(\omega_{L}\right)-N_{R}\left(\omega_{R}\right)\right] .
$$

The averaged cooling efficiency $\eta \equiv-\left\langle J_{R}\right\rangle_{\epsilon} /\langle W\rangle_{\epsilon}$ (the minus emerges due to the sign notation) then becomes

$$
\eta=\frac{\omega_{R}}{\delta \omega}<\frac{T_{R}}{\delta T} \equiv \eta_{\max }
$$

where the inequality was obtained using (17). Thus, the maximal efficiency of our cooling device is exactly given by the Carnot efficiency, reached when the device works reversibly. This result is independent of system properties (spin vs. harmonic mode) and the details of the noise lineshape. Finally, we verify that the average entropy production per unit time is always positive for an irreversible device, $\langle\sigma\rangle_{\epsilon}=$ $\mathcal{T}\left(\frac{\omega_{L}}{T_{L}}-\frac{\omega_{R}}{T_{R}}\right)\left[N_{R}\left(\omega_{R}\right)-N_{L}\left(\omega_{L}\right)\right]$.

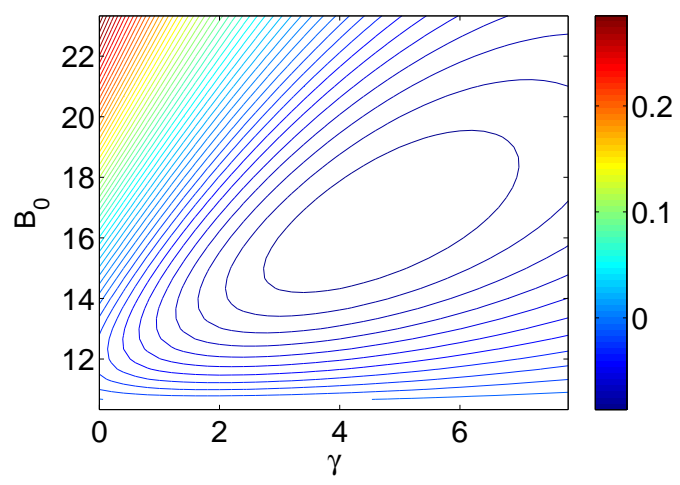

FIG. 2: Color-map of the heat flux at the $R$ contact, revealing a pumping regime (central oval); $\omega_{L}=200, \omega_{R}=3, T_{L}=$ $T_{R}=25$. For these parameters $\left\langle J_{L}\right\rangle_{\epsilon}$ (not plotted) is negative.

Numerical results. We exemplify the operation of the heat pump by simulating Eq. (13) with the stationary 


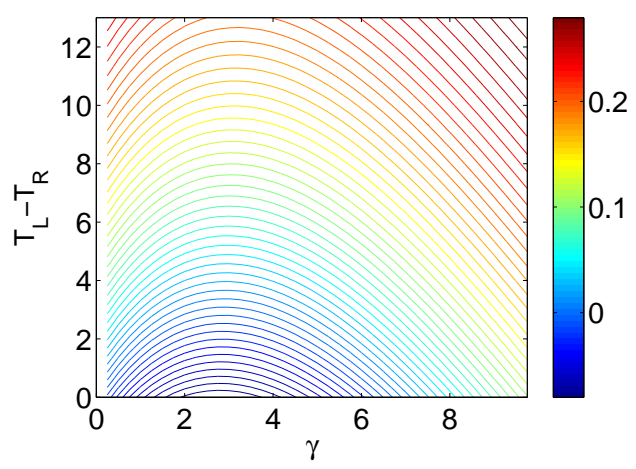

FIG. 3: A color-map of the heat flux at the $R$ contact where $\left\langle J_{R}\right\rangle_{\epsilon}$ is negative for $\gamma<8$ and $T_{L}-T_{R} \lesssim 4$. $\omega_{L}=200$, $\omega_{R}=3, B_{0}=15, T_{R}=25$.

population determined by solving (7) in steady-state. We adopt the Lorentzian lineshape (10), and assume that both solids are characterized by the ohmic spectral function $g_{\nu}(\omega)=A_{\nu} \omega e^{-\omega / \omega_{\nu}}$. We take $\omega_{L} \sim 100$ and $\omega_{R} \sim B_{0} \sim 10, A_{L, R}=1$. The calculations are performed in dimensionless units. If the energy parameters are given in meV, the current divided by the factor $\hbar=4.14$ (meV ps) yields fluxes in units of $\mathrm{meV} / \mathrm{ps}$.

First we assume that $T_{L}=T_{R}$, and demonstrate a unidirectional heat flow from $R$ to $L$. Fig. 2 presents a color-map of the heat current at the $R$ side. The flux at the central oval $\left(\gamma>2 ; 12<B_{0}<20\right)$ is negative, i.e. the $R$ bath is cooled down. We can explain this behavior as follows. For the ohmic function with $\omega_{R}=3$ the $R$ bath is practically disconnected from the TLS at energies of the order $B(t) \gtrsim 15$. Therefore, for $B_{0} \sim 15$ fluctuations that reduce the gap lead to an overlap with the $R$ phonon spectral function, and the TLS can accept energy from this contact. Fluctuations that significantly increase the gap $(B(t)>15)$ imply a complete isolating of the $R$ contact, as its spectral function does not overlap with the molecular vibrational frequency. Energy is then solely exchanged between the (hot) TLS and the $L$ solid, resulting in a net heat flow.

We further demonstrate pumping of heat against a temperature gradient. Fig. 3 shows that $\left\langle J_{R}\right\rangle_{\epsilon}$ is negative for $\gamma<8$ and $T_{L}-T_{R} \lesssim 4$. We verified that the total entropy of the system is increasing. Note that the pumped current is rather small, yet the object of this calculation is to demonstrate the ubiquity of the pumping effect, rather than to develop optimization schemes.

In summary, nano-objects may pump heat effectively by exploiting random fluctuations, given that the (distinct) solids are characterized by frequency dependent spectral functions. Our work reverses standard perceptions of microscopic heat pumps: (i) Directing heat at $\delta T=0$, and pumping heat at finite $\delta T$ are facile processes, taking place in response to a general noise lineshape. (ii) Instead of carefully shaping the external pulse operating on the subsystem, simple bath "shaping" (mode filtering) may lead to maximal performance.

Temperature reduction of nano-level objects is important, e.g. for cooling electronics devices, and for controlling chemical reactions and molecular dynamics. The pumping mechanism described here is pertinent to other systems, as one could realize an analogous exciton heat pump [21]. The effect might be observed in various systems suffering random noise, for example, in nanomechanical resonators. Considering a double clamped nano beam, the natural frequency could be mechanically modulated due to the beam's mass variation resulting from adsorption-desorption processes [22]. The resonator frequency could be also electrostatically tuned by (stochastically) varying the bias applied to a gate electrode [23].

Acknowledgments The work was supported by NSERC and by the University of Toronto Start-up Fund.
[1] P. Reimann, Phys. Rep. 361, 57 (2002).

[2] A. E. Allahverdyan and Th. M. Nieuwenhuizen Phys. Rev. Lett. 85, 232 (2000).

[3] N. Nakagawa and T. Komatsu, Europhys. Lett. 75, 22 (2006).

[4] R. Marathe, A. M. Jayannavar, and A. Dhar, Phys. Rev. E 75, 030103(R) (2007).

[5] E. Geva and R. Kosloff, J. Chem. Phys. 104, 7681 (1996); T. Feldmann, E. Geva, R. Kosloff, and P. Salamon, Am. J. of Phys. 64, 485 (1996).

[6] T. E. Humphrey, R. Newbury, R. P. Taylor, and H. Linke Phys. Rev. Lett. 89, 116801 (2002).

[7] H. T. Quan, Y.-X. Liu, C. P. Sun, and F. Nori, Phys. Rev. E 76, 031105 (2007).

[8] A. E. Allahverdyan, R. S. Johal, and G. Mahler, Phys. Rev. E 77, 041118 (2008).

[9] M. J. Henrich, G. Mahler, and M. Michel, Phys. Rev. E 75, 051118 (2007).
[10] D. Segal and A. Nitzan, Phys. Rev. E 73, 026109 (2006).

[11] M. Rey, M. Strass, S. Kohler, P. Hänggi, and F. Sols, Phys. Rev. B 76, 085337 (2007).

[12] J. P. Pekola and F. W. J. Hekking, Phys. Rev. Lett. 98, 210604 (2007).

[13] R. D. Astumian et al., Proc. Natl. Acad. Sci. 84, 434 (1987).

[14] R. Kubo, J. Math. Phys. 4, 174 (1963).

[15] D. Segal, Phys. Rev. B 73, 205415 (2006).

[16] E. G. Petrov, V. I. Teslenko and I. A. Goychuk, Phys. Rev. E 49, 3894 (1994); I. Goychuk and P. Hanggi, Adv. Phys. 54, 525 (2005).

[17] J. S. Bader and B. J. Berne, J. Chem. Phys. 100, 8359 (1994).

[18] L. Wu and D. Segal, arXiv:0804.3371 1.

[19] V. P. Carey et al., Nanoscale and Microscale Thermophysical Engineering 12,1 (2008).

[20] The random field does not invert the levels, i.e. $E_{1}(t)>$ 
$E_{0}(t)$

[21] D. Segal, Phys. Rev. Lett. 100, 105901 (2008).

[22] Y. T. Yang, et al. Nano Lett. 6, 583 (2006).
[23] V. Sazonova, et al. Nature 431, 284 (2004). 